\title{
Overexpression of an LaeA-like methyltransferase upregulates secondary metabolite production in Aspergillus nidulans
}

Michelle F. Grau ${ }^{\dagger}$, Ruth Entwistle ${ }^{\ddagger}$, C. Elizabeth Oakley ${ }^{\ddagger}$, Clay C. C. Wang ${ }^{+\dagger}$, Berl R. Oakley*‡

${ }^{\dagger}$ Department of Pharmacology and Pharmaceutical Sciences, School of Pharmacy, University of Southern California, Los Angeles, California 90089, United States

${ }^{\ddagger}$ Department of Molecular Biosciences, University of Kansas, Lawrence, Kansas 66045, United States

${ }^{\perp}$ Department of Chemistry, University of Southern California, Dornsife College of Letters, Arts, and Sciences, Los Angeles, California 90089, United States 


\section{Table of Contents}

Detailed Structural Characterization of Compound 23

Compound 23 Spectral Data

Supplemental Figure 1. HPLC paired profile scans of IImG overexpression and mcrA deletion strains compared to the control strain in $\mathrm{GMM}(\mathrm{I})$ and $\mathrm{YG}$.

Supplemental Table 1. Fold change values of secondary metabolites produced by IImG overexpression and $m c r A$ deletion strains compared to the control strain in $G M M(s)$.

Supplemental Table 2. Fold change values of secondary metabolites produced by IImG overexpression and mcrA deletion strains compared to the control strain in YAG.

Supplemental Table 3. Fold change values of secondary metabolites produced by IImG overexpression and mcrA deletion strains compared to the control strain in GMM(I).

Supplemental Table 4. Fold change values of secondary metabolites produced by IImG overexpression and mcrA deletion strains compared to the control strain in YG.

Supplemental Figure 2. UV-Vis and ESIMS (positive or negative mode) spectra of new and unknown compounds identified in this study.

S9-S12

Supplemental Table 5. Primers used in this study.

S13

Supplemental Figure 3. ${ }^{1} \mathrm{H}$ NMR spectrum of 7-methoxy-1-methyl-1,3-dihydroisobenzofuran-1,5-diol (23) in $\mathrm{CD}_{3} \mathrm{OD}(400 \mathrm{MHz})$.

Supplemental Figure 4. ${ }^{13} \mathrm{C}$ NMR spectrum of 7-methoxy-1-methyl-1,3-dihydroisobenzofuran-1,5-diol (23) in $\mathrm{CD}_{3} \mathrm{OD}(400 \mathrm{MHz})$.

S15

Supplemental Figure 5. HMBC correlations $(H \rightarrow C)$ of compound 23.

Supplemental Table 5. NMR spectroscopic data (400 MHz, $\mathrm{CD}_{3} \mathrm{OD}$ ) for compound 23.

Supplemental Figure 6. Proposed cichorine biosynthesis including compounds 22-23, 41 and 47. 


\section{Detailed Structural Characterization of Compound 23}

Compound $\mathbf{2 3}$ was isolated as a white amorphous powder. The molecular formula was found to be $\mathrm{C}_{10} \mathrm{H}_{12} \mathrm{O}_{4}$ by its ${ }^{1} \mathrm{H}$ NMR, ${ }^{13} \mathrm{C}$ NMR (Supplemental Figures 3 and 4 ) and HRESIMS spectral data, representing five indices of hydrogen deficiency (IHD). ${ }^{1} \mathrm{H},{ }^{13} \mathrm{C}$, gHSQC NMR spectra indicated compound 2 contains one methyl group $\left[\delta_{H} 2.06(3 \mathrm{H}, \mathrm{s}), \delta_{C} 8.9\right]$, one methoxy group [ $\left.\delta_{H} 3.86(3 \mathrm{H}, \mathrm{s}), \delta_{\mathrm{C}} 60.3\right]$, one oxymethylene group $\left[\delta_{H} 4.84-4.89(1 \mathrm{H})\right.$ and $\left.5.02(1 \mathrm{H}, \mathrm{br} \mathrm{d}, J=12.5 \mathrm{~Hz}), \delta_{\mathrm{C}} 72.9\right]$, two tertiary olefinic or hemiacetal moieties $\left[\delta_{H} 6.27(1 \mathrm{H}, \mathrm{s}), \delta_{\mathrm{C}} 108.0\right.$ and $\left.\delta_{\mathrm{H}} 6.43(1 \mathrm{H}, \mathrm{s}), \delta_{\mathrm{c}} 102.6\right]$ and five quaternary carbons $\left(\delta_{c} 116.5,119.5,141.3,155.9\right.$ and 159.4$)$ that exhibit chemical shifts in the aromatic region. Assuming that six of these carbons belong to an aromatic ring accounts for four IHD, while the last IHD must be due to the presence of a second ring since no alkene, aldehyde or ester carbons were detected by NMR. The two most downfield shifted aromatic carbons $\left(\delta_{c} 155.9\right.$ and 159.4$)$ are attached to a hydroxyl and the methoxy group accounting for two of the four oxygens. Because of the downfield shift of the methyl group $\left[\delta_{H} 2.06(3 \mathrm{H}, \mathrm{s}), \delta_{\mathrm{C}} 8.9\right]$ we predicted it to be a substituent of the aromatic ring. With the second ring forming attachments to two of the aromatic carbons, it leaves one of the tertiary carbons $\left[\delta_{H} 6.27\right.$ $(1 \mathrm{H}, \mathrm{s}), \delta_{\mathrm{C}} 108.0$ and $\left.\delta_{\mathrm{H}} 6.43(1 \mathrm{H}, \mathrm{s}), \delta_{\mathrm{C}} 102.6\right]$ to belong to the aromatic ring, and the other to belong to the second ring. The penta-substituted aromatic ring could be assigned unambiguously by the long-rang $1 \mathrm{H}-13 \mathrm{C}$ gHMBC correlations (Supplemental Figure 5) The remainder of the two carbons $\left(\delta_{\mathrm{C}} 72.9 \mathrm{CH}_{2}\right.$ and $108.0, \mathrm{CH}$ ) on the second ring was attributed to an oxymethylene and a hemiacetal functional groups. gHSQC and long-range gHMBC correlations (Supplemental Figure 5) allowed the full assignment of the structure (Supplemental Table 5).

\section{Compound 23 Spectral Data}

7-methoxy-6-methyl-1,3-dihydroisobenzofuran-1,5-diol (23): white powder; UV $\lambda_{\max }{ }^{\mathrm{MeOH}} \mathrm{nm}$ : 233, 281; ${ }^{1} \mathrm{H}$ $\operatorname{NMR}\left(\mathrm{CD}_{3} \mathrm{OD}\right): \delta=2.06(3 \mathrm{H}, \mathrm{s}), 3.86(3 \mathrm{H}, \mathrm{s}), 4.94(2 \mathrm{H}, \mathrm{dddq}), 6.27(1 \mathrm{H}, \mathrm{d}), 6.43(1 \mathrm{H}, \mathrm{s}) ;{ }^{13} \mathrm{C} \mathrm{NMR}\left(\mathrm{CD}_{3} \mathrm{OD}\right)$ : $\delta=8.9,60.3,72.9,102.6,108.0,116.5,119.5,141.3,155.9,159.4$. For UV and ESIMS spectrum, see Supplemental Figure 2; HRESIMS obtained $m / z[M-H]=195.0654$ (calcd 195.0657 for $\mathrm{C}_{10} \mathrm{H}_{11} \mathrm{O}_{4}$ ). 


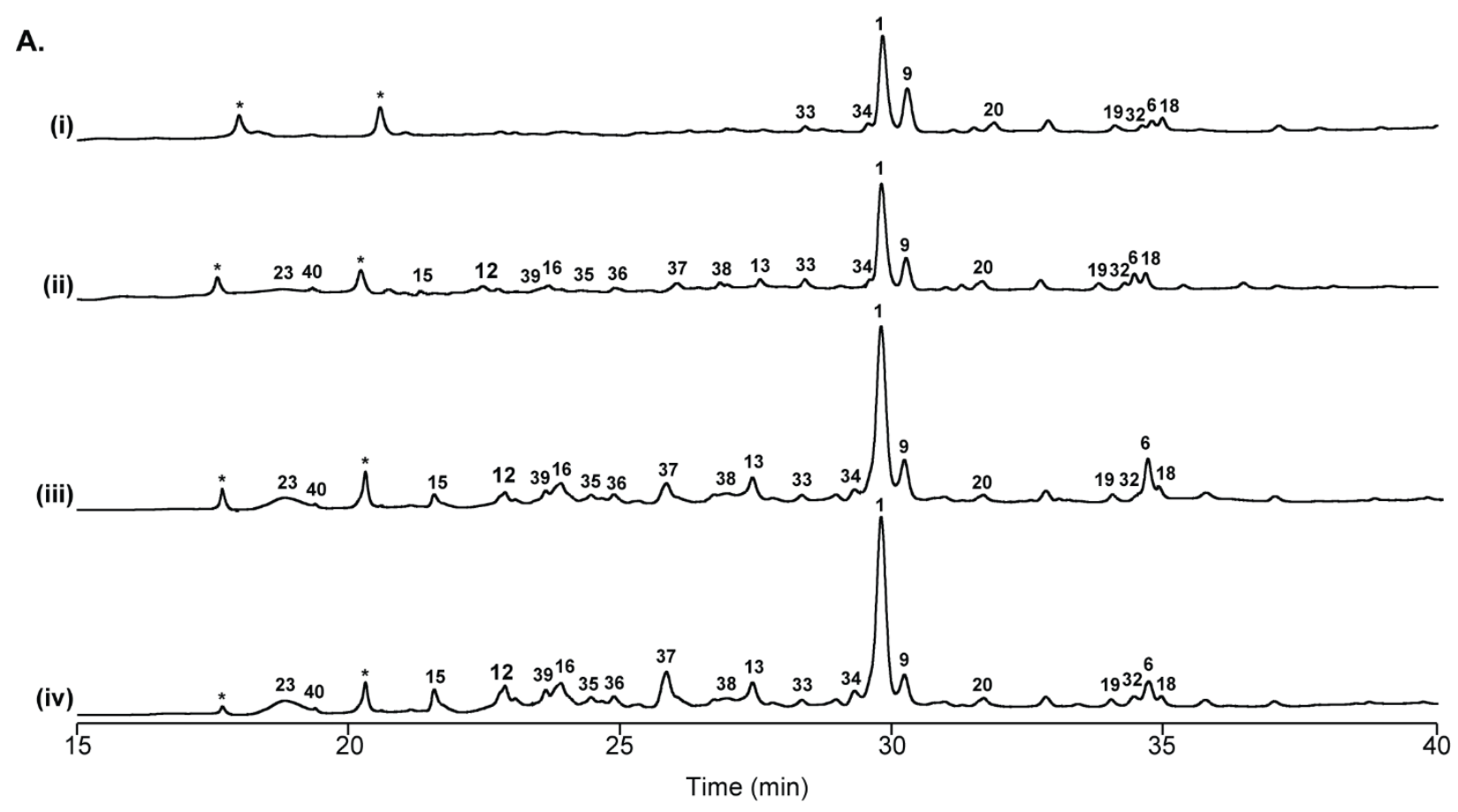

B.
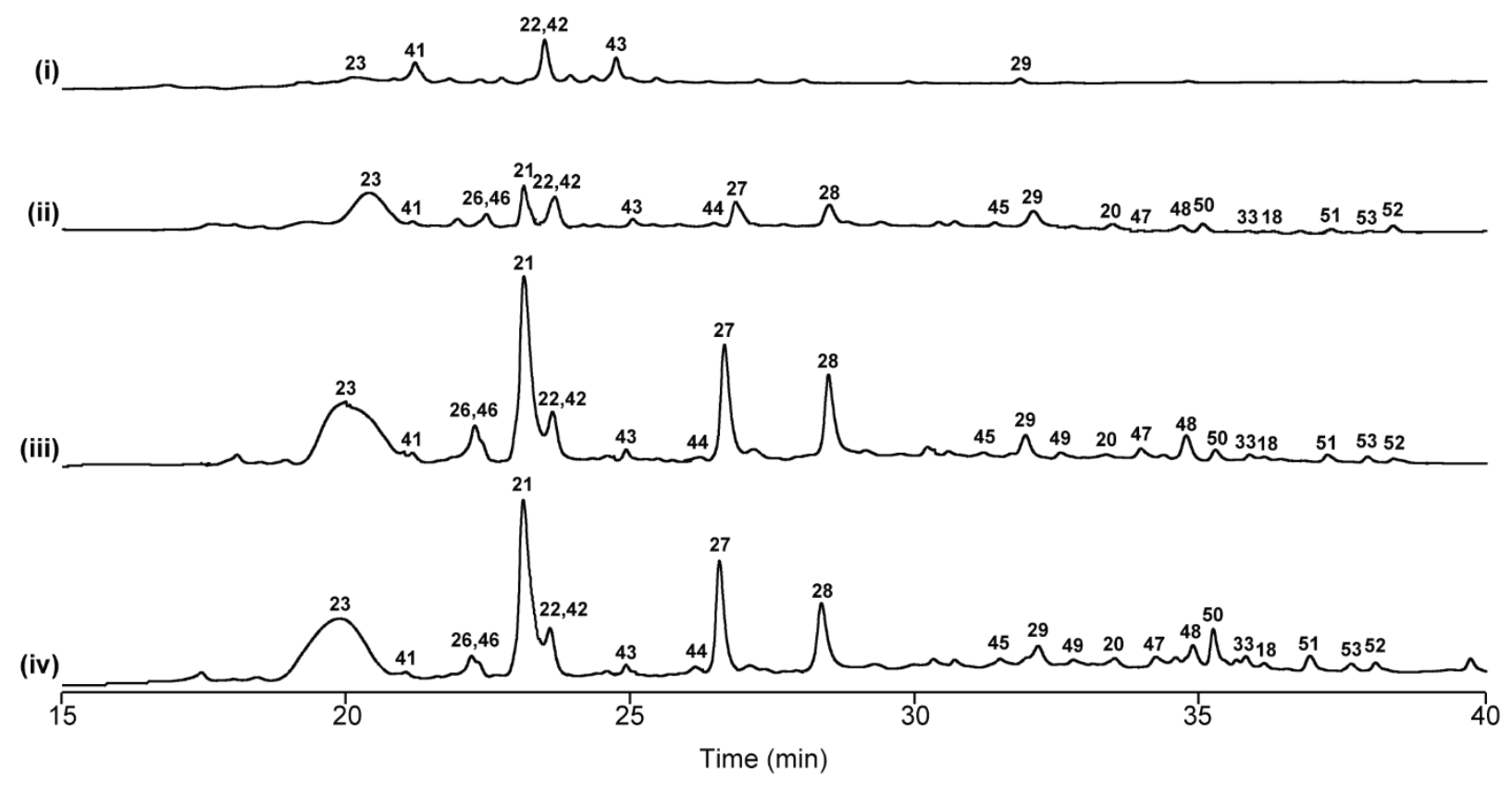

Supplemental Figure 1. HPLC paired profile scans of IImG overexpression and mcrA deletion strains compared to the control strain in liquid media. A. GMM(I) and B. YG. (i) $/ 1 m G^{+}, m c r A^{+}$ (LO11174), (ii) gpdA(p)/lmG, $m c r A^{+}$(LO10860), (iii) IlmG ${ }^{+}, m c r A \Delta$ (LO11177), and (iv) $\operatorname{gpd} A(\mathrm{p}) / / m G, \operatorname{mcr} A$ (LO10881). 
Supplemental Table 1. Fold change values of secondary metabolites produced by IImG overexpression and $m c r A$ deletion strains compared to the control strain LO11174 (//mG $\left.G^{+}, m c r A^{+}\right)$in GMM(s). LO10860 = $\operatorname{gpd} A(\mathrm{p}) / / m G, m c r A^{+} ; \mathrm{LO} 10864=\mathrm{hnmtA}(\mathrm{p}) / / m G, m c r A^{+} ; \mathrm{LO} 11177=\| / m G^{+}, m c r A \Delta ; \mathrm{LO} 10868=$ $\mathrm{hnmtA}(\mathrm{p}) / / m G, m c r A \Delta ; \mathrm{LO} 10881=g p d A(\mathrm{p}) / / m G, m c r A \Delta$.

\begin{tabular}{|c|c|c|c|c|c|c|}
\hline \multicolumn{7}{|c|}{ GMM(s) } \\
\hline \multirow{2}{*}{ Pathway } & \multirow{2}{*}{ Compound } & \multicolumn{5}{|c|}{ Fold $\Delta$ from L011174 } \\
\hline & & LO10860 & L010864 & L011177 & L010868 & LO10881 \\
\hline Sterigmatocystin & Sterigmatocystin (1) & 2.41 & 2.22 & 4.00 & 5.00 & 5.97 \\
\hline Sterigmatocystin & 3'-hydroxyversiconol (11) & 2.55 & 2.26 & 3.93 & 4.41 & 4.19 \\
\hline Sterigmatocystin & Versiconol (12) & 1.52 & 1.26 & 3.65 & 4.16 & 4.49 \\
\hline Sterigmatocystin & Nidurufin (13) & 1.05 & 0.50 & 3.59 & 4.51 & 5.45 \\
\hline Sterigmatocystin & Averufin (14) & 3.30 & 3.65 & 11.83 & 14.83 & 15.03 \\
\hline Sterigmatocystin & Unknown (15) & 1.85 & 1.68 & 5.95 & 6.87 & 7.39 \\
\hline Sterigmatocystin & Unknown (16) & 2.10 & 1.66 & 5.26 & 6.36 & 8.69 \\
\hline Sterigmatocystin & Unknown (17) & 1.50 & 1.40 & 4.19 & 4.95 & 5.28 \\
\hline Austinol & Neoaustinone (2) & 1.37 & 1.32 & 1.06 & 1.12 & 1.31 \\
\hline Austinol & Austinol (3) & 1.26 & 1.20 & 1.22 & 1.29 & 1.57 \\
\hline Austinol & Dehydroaustinol (4) & 1.32 & 1.25 & 1.37 & 1.34 & 1.48 \\
\hline Austinol & Austinolide (5) & 1.15 & 1.16 & 1.21 & 1.31 & 1.33 \\
\hline Terrequinone & Terrequinone A (6) & 1.12 & 1.16 & 2.81 & 3.83 & 3.70 \\
\hline Prenyl Xanthone & Emericellin (7) & 4.56 & 5.58 & 93.24 & 111.60 & 124.21 \\
\hline Unknown & Unknown (8) & 1.12 & 0.74 & 1.38 & 1.22 & 1.60 \\
\hline Unknown & Unknown (9) & 1.88 & 1.75 & 1.65 & 1.70 & 1.72 \\
\hline Unknown & Unknown (10) & 1.42 & 1.34 & 0.20 & 0.20 & 0.35 \\
\hline Nidulanin & Nidulanin A (18) & 1.40 & 1.31 & 3.04 & 3.61 & 4.83 \\
\hline Nidulanin & Nidulanin A analog (19) & 1.50 & 1.42 & 3.39 & 3.75 & 4.40 \\
\hline Nidulanin & Nidulanin A + O (20) & 1.74 & 1.42 & 3.77 & 4.57 & 5.49 \\
\hline
\end{tabular}


Supplemental Table 2. Fold change values of secondary metabolites produced by IImG overexpression and $m c r A$ deletion strains compared to the control strain L011174 $\left(/ / m G^{+}, m c r A^{+}\right)$in YAG. LO10860 = $\operatorname{gpdA}(\mathrm{p}) / / m G, m c r A^{+} ; \mathrm{LO} 10864=\mathrm{hnmtA}(\mathrm{p}) / / m G, m c r A^{+} ;$LO11177 = IImG ${ }^{+}, m c r A \Delta ;$ LO10868 = hnmtA(p)/ImG, $m c r A \Delta ;$ LO10881 = gpdA(p)/ImG, mcrA $\Delta$.

\begin{tabular}{|l|l|c|c|c|c|c|}
\hline \multicolumn{3}{|c|}{ YAG } \\
\hline \multirow{2}{*}{ Pathway } & \multicolumn{2}{|c|}{ Compound } & \multicolumn{4}{c|}{ Fold $\Delta$ from LO11174 } \\
\cline { 3 - 7 } & & LO10860 & L010864 & LO11177 & LO10868 & L010881 \\
\hline Sterigmatocystin & Sterigmatocystin (1) & 15.11 & 12.98 & 39.78 & 55.63 & 37.59 \\
\hline Sterigmatocystin & Averufin (14) & 4.55 & 3.32 & 4.62 & 6.04 & 6.11 \\
\hline Sterigmatocystin & Versicolorin B (24) & 24.74 & 21.33 & 37.46 & 45.94 & 39.51 \\
\hline Sterigmatocystin & Averantin (25) & 4.42 & 3.93 & 6.32 & 8.31 & 7.62 \\
\hline Monodictyphenone/Emodin & 2,w-dihydroxyemodin (21) & 1.64 & 1.21 & 3.66 & 2.93 & 2.14 \\
\hline Monodictyphenone/Emodin & Monodictyphenone (26) & 3.86 & 3.86 & 13.34 & 5.84 & 5.57 \\
\hline Monodictyphenone/Emodin & w-hydroxyemodin (27) & 15.62 & 11.10 & 37.39 & 46.54 & 39.33 \\
\hline Monodictyphenone/Emodin & 2-hydroxyemodin (28) & 8.46 & 8.02 & 13.98 & 14.90 & 13.49 \\
\hline Monodictyphenone/Emodin & Emodin (29) & 3.87 & 3.63 & 6.25 & 7.35 & 7.11 \\
\hline Monodictyphenone/Emodin & Chrysophanol (30) & 3.68 & 4.43 & 5.43 & 6.50 & 6.75 \\
\hline Nidulanin & Nidulanin A (18) & 2.02 & 1.29 & 7.05 & 11.50 & 13.22 \\
\hline Nidulanin & Nidulanin A analog (19) & 3.08 & 2.11 & 11.94 & 21.75 & 23.86 \\
\hline Cichorine & 3-methylorsellinic acid (22) & 1.78 & 1.31 & 2.01 & 2.36 & 2.96 \\
\hline Cichorine & Cichorine intermediate (23) & 3.09 & 2.93 & 4.28 & 4.06 & 3.98 \\
\hline Unknown & Unknown (31) & 1.66 & 1.45 & 5.86 & 3.06 & 2.39 \\
\hline
\end{tabular}


Supplemental Table 3. Fold change values of secondary metabolites produced by IImG overexpression and $m c r A$ deletion strains compared to the control strain L011174 $\left(/ / m G^{+}, m c r A^{+}\right)$in $\mathrm{GMM}(\mathrm{I})$. LO10860 =

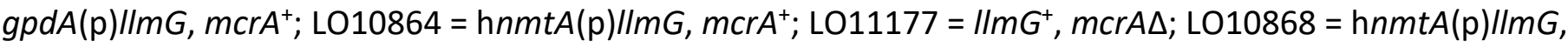
mcrA $;$; LO10881 = gpdA(p)/lmG, mcrA $\Delta$.

\begin{tabular}{|c|c|c|c|c|c|c|}
\hline \multicolumn{7}{|c|}{ GMM(I) } \\
\hline \multirow{2}{*}{ Pathway } & \multirow{2}{*}{ Compound } & \multicolumn{5}{|c|}{ Fold $\Delta$ from L011174 } \\
\hline & & LO10860 & LO10864 & L011177 & L010868 & L010881 \\
\hline Sterigmatocystin & Sterigmatocystin (1) & 96.32 & 101.29 & 100.10 & 190.12 & 219.35 \\
\hline Sterigmatocystin & Versiconol (12) & 1.81 & 1.45 & 2.89 & 6.03 & 6.02 \\
\hline Sterigmatocystin & Nidurufin (13) & 6.18 & 5.75 & 7.83 & 11.32 & 14.47 \\
\hline Sterigmatocystin & Unknown (15) & 2.92 & 2.22 & 4.00 & 9.01 & 8.04 \\
\hline Sterigmatocystin & Unknown (16) & 5.15 & 2.59 & 5.56 & 12.91 & 8.58 \\
\hline Sterigmatocystin & Unknown (35) & 1.77 & 1.60 & 10.21 & 18.27 & 20.50 \\
\hline Sterigmatocystin & Unknown (36) & 1.81 & 1.59 & 2.61 & 4.41 & 4.64 \\
\hline Sterigmatocystin & Unknown (37) & 3.45 & 2.71 & 7.59 & 9.36 & 12.14 \\
\hline Sterigmatocystin & Unknown (38) & 1.92 & 1.49 & 2.45 & 2.85 & 2.54 \\
\hline Terrequinone & Terrequinone A (6) & 11.98 & 8.81 & 26.39 & 28.75 & 42.33 \\
\hline Nidulanin & Nidulanin A (18) & 1.93 & 1.65 & 1.38 & 2.52 & 1.09 \\
\hline Nidulanin & Nidulanin A analog (19) & 0.93 & 1.07 & 0.90 & 1.65 & 1.24 \\
\hline Nidulanin & Nidulanin A + O (20) & 1.47 & 2.77 & 1.03 & 1.60 & 0.98 \\
\hline Nidulanin & Nidulanin A analog (32) & 1.40 & 1.37 & 1.34 & 2.50 & 1.31 \\
\hline Cichorine & Cichorine Intermediate (23) & 1.29 & 1.44 & 18.02 & 23.94 & 28.66 \\
\hline Cichorine & Cichorine (40) & 2.06 & 2.19 & 2.63 & 5.20 & 4.00 \\
\hline Cichorine & $\begin{array}{l}\text { O-Methyl-3-methylorsellinaldehyde } \\
\text { dimer (39) }\end{array}$ & 3.29 & 1.64 & 39.82 & 28.54 & 74.91 \\
\hline Unknown & Unknown (9) & 0.98 & 1.14 & 1.00 & 1.32 & 1.01 \\
\hline Unknown & Unknown (33) & 1.39 & 0.98 & 1.21 & 1.55 & 1.43 \\
\hline Unknown & Unknown (34) & 0.99 & 1.21 & 1.46 & 1.49 & 1.79 \\
\hline
\end{tabular}


Supplemental Table 4. Fold change values of secondary metabolites produced by IImG overexpression and $m c r A$ deletion strains compared to the control strain LO11174 $\left(/ / m G^{+}, m c r A^{+}\right)$in YG. LO10860 $=g p d A(p) / I m G$,

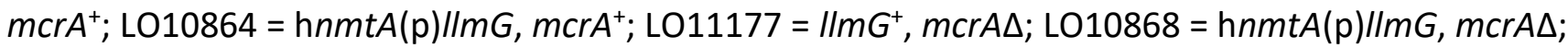
LO10881 $=\operatorname{gpd} A(\mathrm{p}) / / m G, \operatorname{mcr} A$.

\begin{tabular}{|c|c|c|c|c|c|c|}
\hline \multicolumn{7}{|c|}{ YG } \\
\hline \multirow{2}{*}{ Pathway } & \multirow{2}{*}{ Compound } & \multicolumn{5}{|c|}{ Fold $\Delta$ from L011174 } \\
\hline & & LO10860 & L010864 & L011177 & L010868 & LO10881 \\
\hline Monodictyphenone/Emodin & 2,w-dihydroxyemodin (21) & 7.77 & 5.23 & 154.44 & 206.81 & 208.77 \\
\hline Monodictyphenone/Emodin & Monodictyphenone (26) & 4.44 & 3.78 & 18.17 & 28.45 & 32.77 \\
\hline Monodictyphenone/Emodin & $\omega$-hydroxyemodin (27) & 9.29 & 10.37 & 16.78 & 36.72 & 62.89 \\
\hline Monodictyphenone/Emodin & 2-hydroxyemodin (28) & 12.15 & 20.67 & 34.68 & 51.57 & 58.25 \\
\hline Monodictyphenone/Emodin & Emodin (29) & 23.21 & 22.87 & 36.28 & 52.36 & 46.66 \\
\hline Monodictyphenone/Emodin & Atrochrysone (44) & 2.31 & 2.67 & 2.83 & 5.16 & 6.80 \\
\hline Monodictyphenone/Emodin & $\begin{array}{l}\text { (cis/trans)-emodin-physicon } \\
\text { bianthrone (47) }\end{array}$ & 9.71 & 7.92 & 50.87 & 51.89 & 58.20 \\
\hline Monodictyphenone/Emodin & $\begin{array}{l}\text { (cis/trans)-emodin-physicon } \\
\text { bianthrone (48) }\end{array}$ & 4.99 & 4.57 & 66.32 & 89.14 & 82.87 \\
\hline Nidulanin & Nidulanin A (18) & 4.34 & 2.12 & 6.26 & 10.19 & 8.34 \\
\hline Nidulanin & Nidulanin A + O (20) & 4.23 & 2.52 & 7.22 & 7.29 & 8.21 \\
\hline Nidulanin & Nidulanin A analog (32) & 7.04 & 4.11 & 16.45 & 23.38 & 33.29 \\
\hline Nidulanin & Nidulanin A - prenyl (45) & 3.31 & 2.43 & 4.32 & 4.80 & 5.77 \\
\hline F-9775A/F-9775B & Orsellinic acid (41) & 1.36 & 3.07 & 2.07 & 1.91 & 2.68 \\
\hline F-9775A/F-9775B & F-9775A (42) & 2.16 & 2.55 & 1.32 & 1.26 & 1.85 \\
\hline F-9775A/F-9775B & F-9775B (43) & 1.07 & 1.89 & 1.45 & 1.40 & 1.97 \\
\hline Cichorine & 3-methylorsellinic acid (22) & 4.16 & 3.49 & 5.77 & 7.58 & 9.09 \\
\hline Cichorine & Cichorine Intermediate (23) & 4.64 & 4.16 & 9.23 & 12.36 & 17.01 \\
\hline Cichorine & Nidulol (46) & 2.50 & 3.52 & 4.66 & 7.45 & 8.94 \\
\hline Unknown & Unknown (49) & 72.17 & 44.55 & 281.04 & 365.93 & 463.65 \\
\hline Unknown & Unknown (50) & 3.53 & 4.46 & 42.87 & 52.12 & 48.40 \\
\hline Unknown & Unknown (51) & 57.65 & 43.96 & 435.18 & 445.75 & 456.49 \\
\hline Unknown & Unknown (52) & 97.78 & 83.08 & 328.77 & 378.11 & 431.57 \\
\hline Unknown & Unknown (53) & 8.45 & 10.81 & 44.46 & 42.99 & 49.28 \\
\hline
\end{tabular}


Supplemental Figure 2. UV-Vis and ESIMS (positive or negative mode) spectra of new and unknown compounds identified in this study.

Unknown (8)
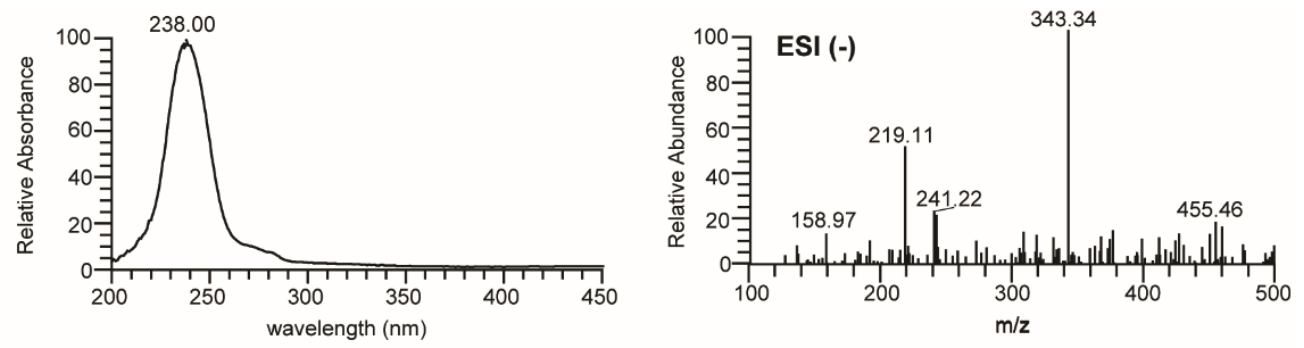

Unknown (9)
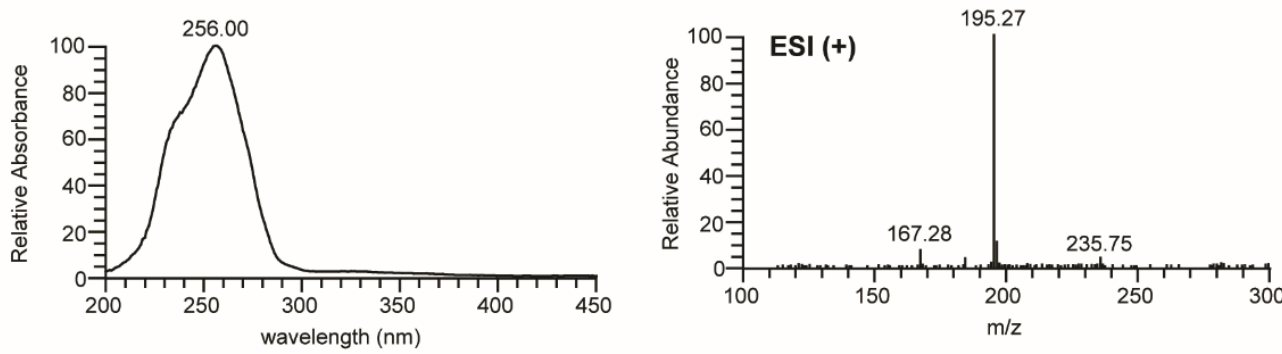

Unknown (9)
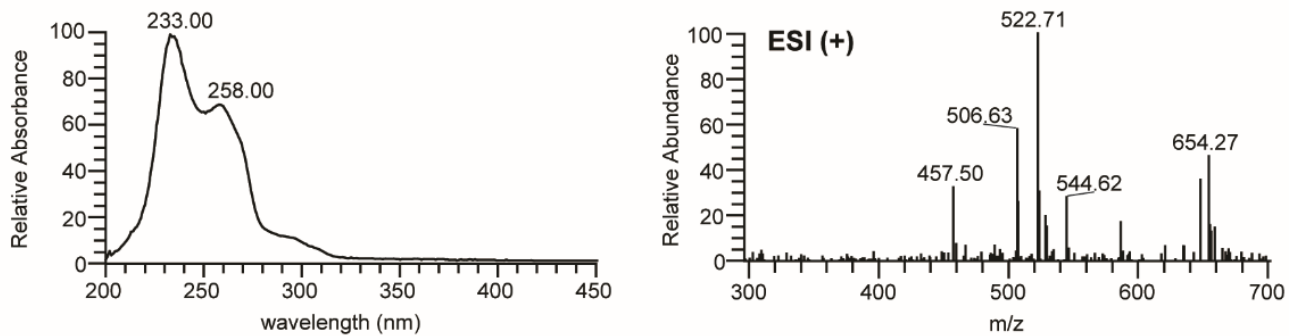

Unknown (15)

(Sterigmatocystin intermediate or shunt product)
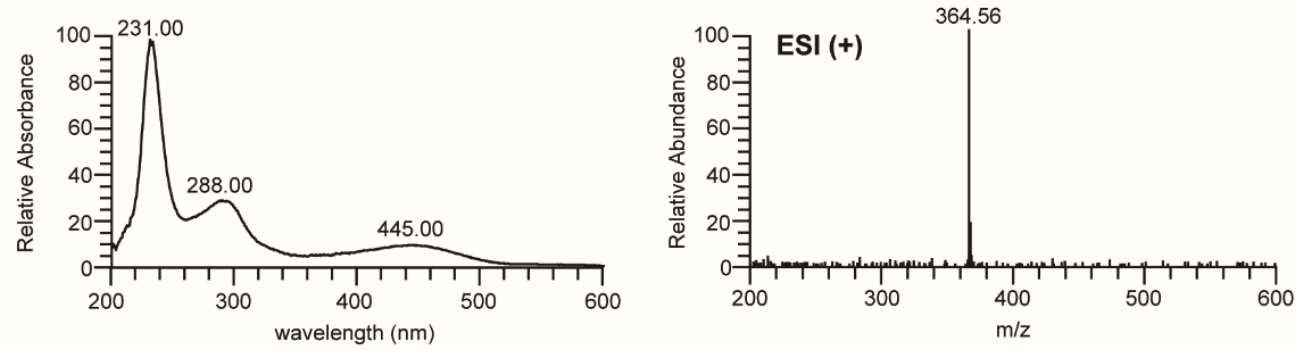

Unknown (16)

(Sterigmatocystin intermediate or shunt product)

Unknown (17)

(Sterigmatocystin intermediate or shunt product)
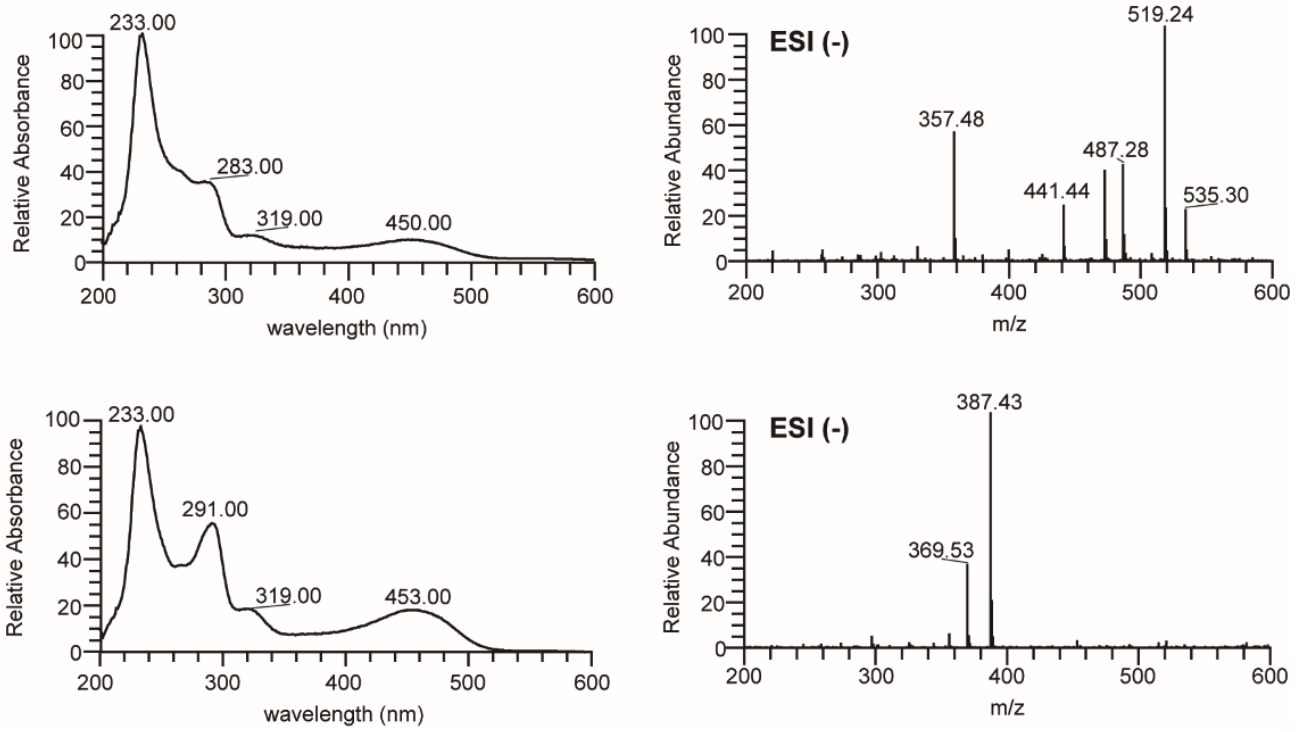
<smiles>COc1c(C)c(O)cc2c1C(O)OC2</smiles>

Unknown (23)

(Cichorine intermediate)

Unknown (31)

Unknown (33)

Unknown (34)

Unknown (35)

(Sterigmatocystin intermediate or shunt product)

Unknown (36)

(Sterigmatocystin intermediate or shunt product)
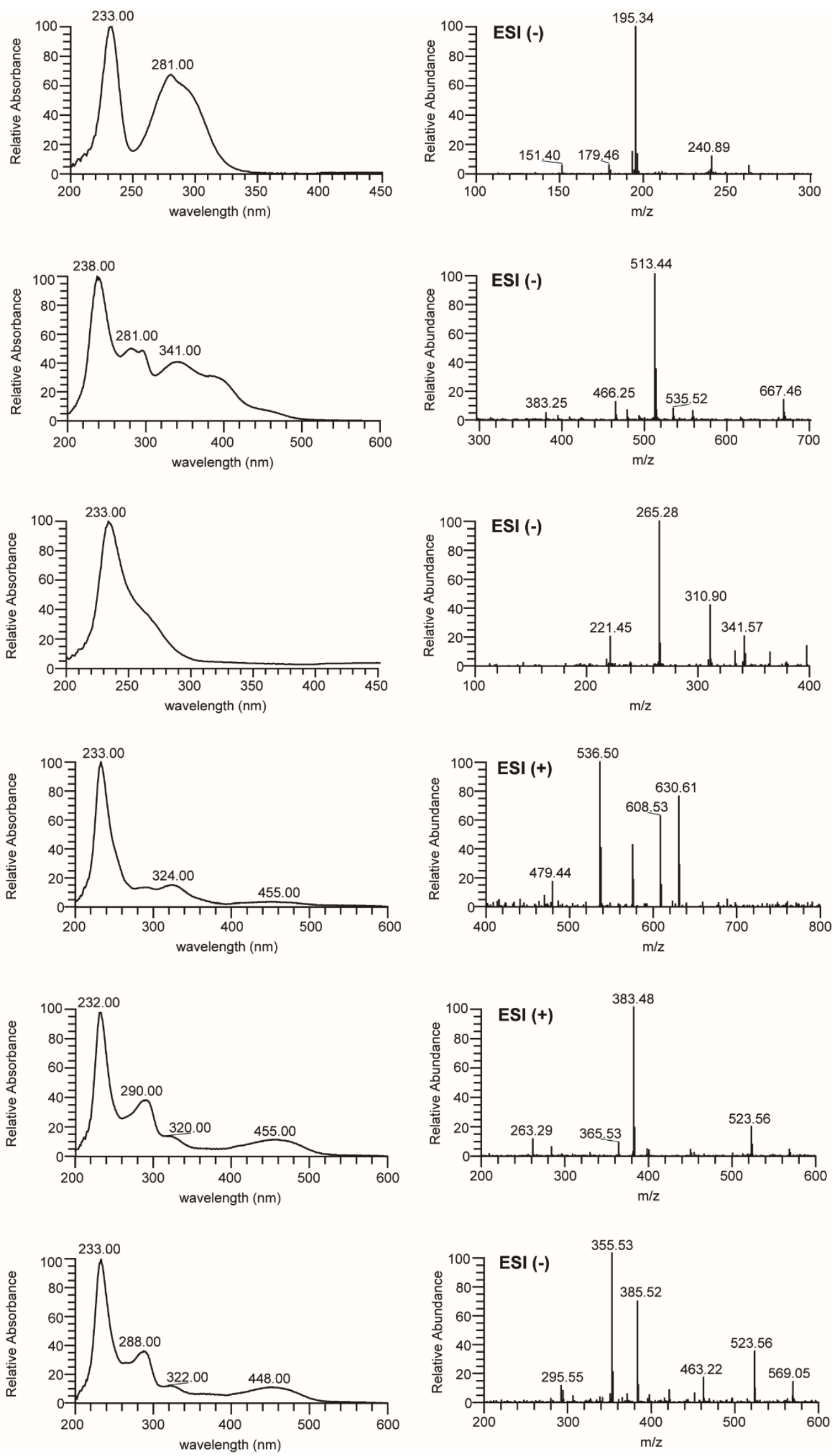
Unknown (37)

(Sterigmatocystin intermediate or shunt product)

Unknown (38)

(Sterigmatocystin intermediate or shunt product)

Unknown (49)

Unknown (50)

Unknown (51)

Unknown (52)
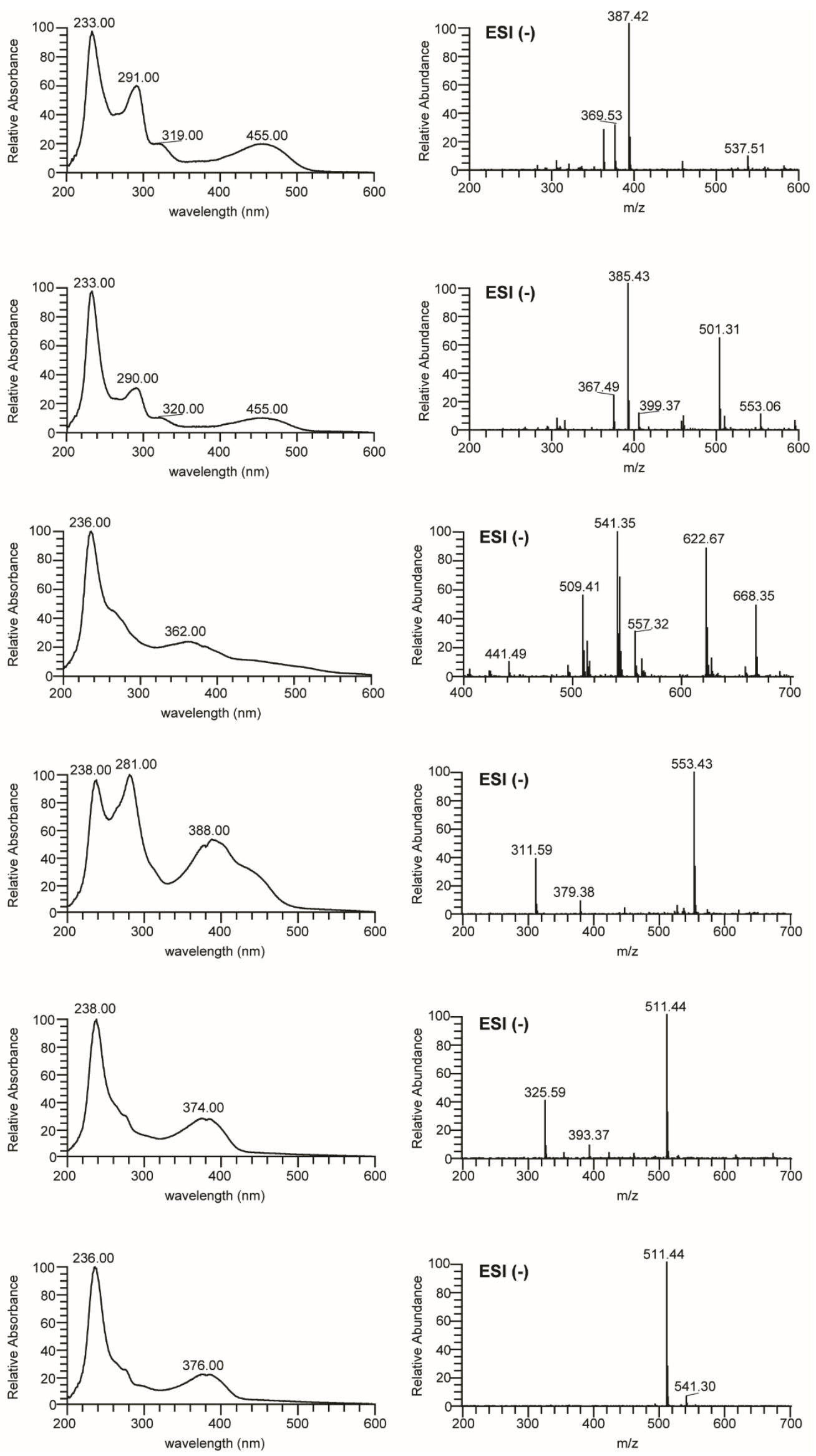
Unknown (53)
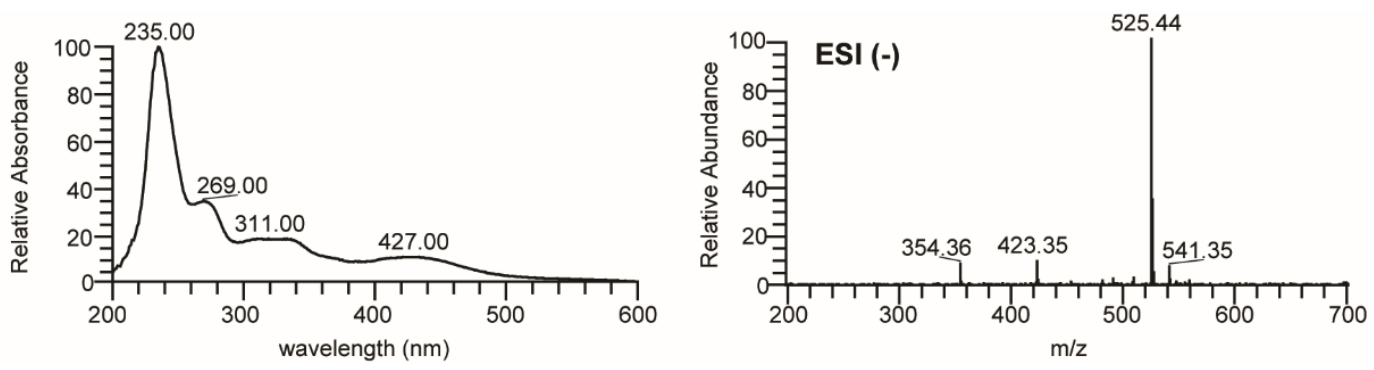
Supplemental Table 5. Primers used in this study.

Note: The lower-case letters designate "tails," i.e. sequences that are not part of the genomic sequences amplified, but used for fusing fragments together.

\begin{tabular}{|l|l|}
\hline LizP2018 & caatgctcttcaccctcttcTGGGCGGGTTCTTTTGGTTT \\
\hline LizP2019 & ctgtctgagaggaggcactACTATCAAGTAGTACGAGTTAC \\
\hline LizP2273 & TGTGATGTCTGCTCAAGCGG \\
\hline LizP4139 & ATGTGCGCCCACTCGGAG \\
\hline LizP4800 & gcatcagtgcctcctctcagacagcCACCTTCAGTGGACTCGAG \\
\hline LizP6398 & CAGAAGCAGTACCATGGCG \\
\hline LizP6399 & CGGCAAGGATGAGCAGGC \\
\hline LizP6852 & CGTCAACTTCGGAACACGG \\
\hline LizP6853 & TCCGGATGGACTAACTGC C \\
\hline LizP6854 & CGTCTTCCGAGAACGTCAG \\
\hline LizP6855 & cgaagagggtgaagagcattgATTGGGCTGGATTAAACTGAC \\
\hline LizP6856 & gcatcagtgcctcctctcagacagCCATATGTTTATTGCAGCCAG \\
\hline LizP6857 & TTATCCGCAGAGCTCTAATTC \\
\hline LizP6858 & AAGACAATGGAGAGGTGACC \\
\hline LizP6859 & CGATGCGATTGTCAAGTGAG \\
\hline LizP7020 & GCTATCCTCAATCTGGCTTG \\
\hline LizP7021 & CCACAAGCTCGTGTCACATC \\
\hline LizP7022 & GTGAACCAAGCACACAGGAG \\
\hline LizP7023 & cgaagagggtgaagagcattgGCTCCATACCTCGAGATCTC \\
\hline LizP7025 & ccgcttgagcagacatcacaATGACGTACGCATCTCTCCAAC \\
\hline LizP7026 & CTCATGAGCTGCGAGCTCC \\
\hline LizP7027 & GGTAGAGGGATCATCTTTGC \\
\hline LizP7028 & CTCTGACTTGTGTTGACGTC \\
\hline
\end{tabular}


Supplemental Figure 3. ${ }^{1} \mathrm{H}$ NMR spectrum of 7-methoxy-1-methyl-1,3-dihydroisobenzofuran-1,5-diol (23) in $\mathrm{CD}_{3} \mathrm{OD}(400 \mathrm{MHz})$.

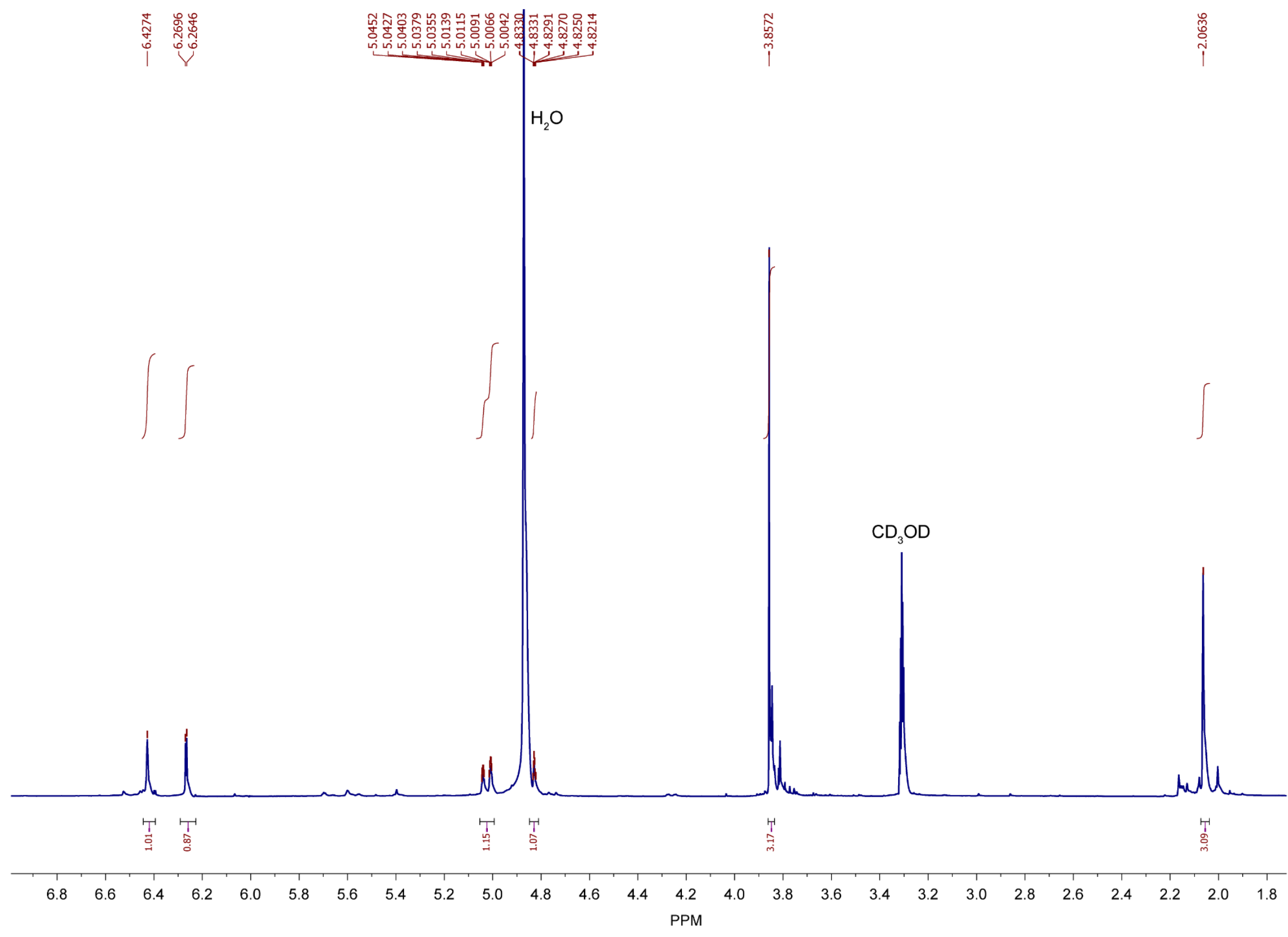


Supplemental Figure 4. ${ }^{13} \mathrm{C}$ NMR spectrum of 7-methoxy-1-methyl-1,3-dihydroisobenzofuran-1,5-diol (23) in $\mathrm{CD}_{3} \mathrm{OD}(400 \mathrm{MHz})$.

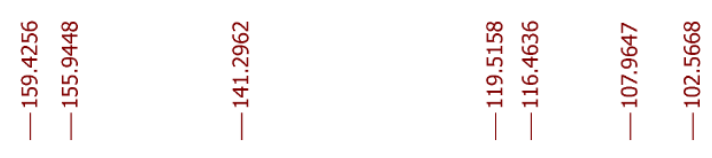$$
\text { ì }
$$
PPM

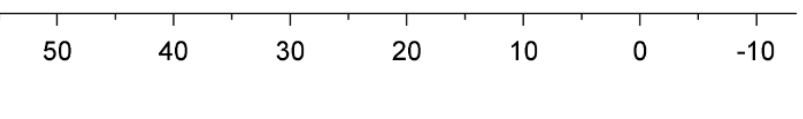




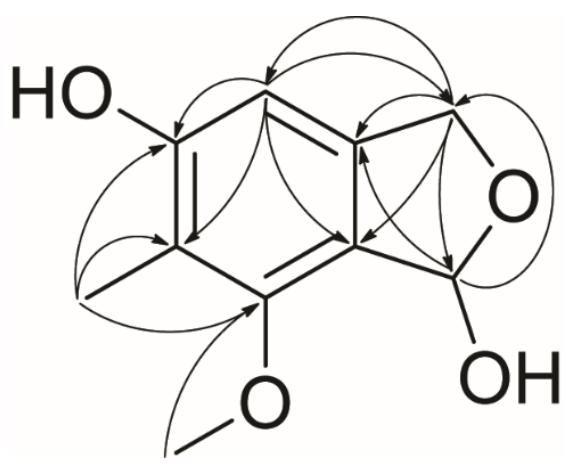

Supplemental Figure 5. HMBC correlations $(\mathrm{H} \rightarrow \mathrm{C})$ of compound 23. 
Supplemental Table 5. NMR spectroscopic data (400 MHz, CD ${ }_{3} \mathrm{OD}$ ) for compound 23.

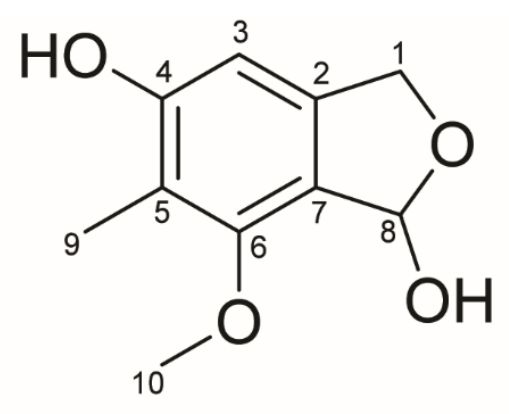

23

\begin{tabular}{ccc}
\hline & \multicolumn{2}{c}{ Compound 23 } \\
\cline { 2 - 3 } Position & $\delta_{\mathrm{C}}$, type & $\delta_{\mathrm{H}},(\mathrm{J}$ in Hz$)$ \\
\hline 1 & $72.9, \mathrm{CH}$ & $4.84-4.89(1 \mathrm{H})^{*}, 5.02(1 \mathrm{H}, \mathrm{br} \mathrm{d}, 12.5)$ \\
2 & $141.3, \mathrm{C}$ & - \\
3 & $102.6, \mathrm{CH}$ & $6.43(1 \mathrm{H}, \mathrm{s})$ \\
4 & $159.4, \mathrm{C}$ & - \\
5 & $116.5, \mathrm{C}$ & - \\
6 & $155.9, \mathrm{C}$ & - \\
7 & $119.5, \mathrm{C}$ & - \\
8 & $108.0, \mathrm{CH}$ & $6.27(1 \mathrm{H}, \mathrm{d}, 1.9)$ \\
9 & $8.9, \mathrm{CH}_{3}$ & $2.06(3 \mathrm{H}, \mathrm{s})$ \\
10 & $60.3, \mathrm{CH}_{3}$ & $3.86(3 \mathrm{H}, \mathrm{s})$ \\
\hline
\end{tabular}

*Signal overlaps with HOD signal 


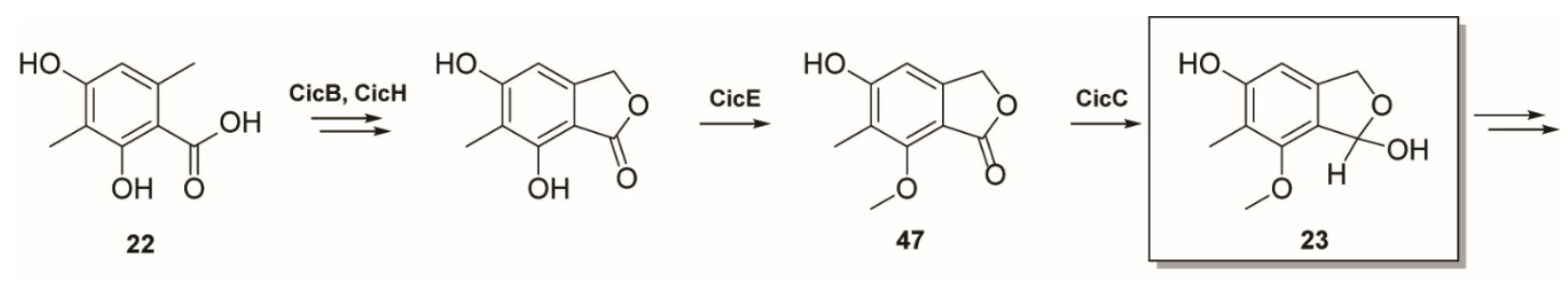<smiles>C=C=Cc1c(C(=O)O)cc(O)c(C)c1OC</smiles>

41

Supplemental Figure 6. Proposed cichorine biosynthesis including compounds 22-23, 41 and 47. 